\title{
The influence of carbon nanotube addition on the properties of shear thickening fluid
}

\author{
PAULINA NAKONIECZNA*, LUKASZ WIERZBICKI, RAFAŁ WRÓBLEWSKI, \\ TOMASZ PŁOCIŃSKI and MARCIN LEONOWICZ \\ Faculty of Materials Science and Engineering, Warsaw University of Technology, Warsaw, Poland \\ *Author for correspondence (Paulina.Nakonieczna.dokt@pw.edu.pl)
}

MS received 16 August 2018; accepted 1 February 2019; published online 21 May 2019

\begin{abstract}
The properties of shear thickening fluid (STF), based on polypropylene glycol and amorphous silica, modified by the addition of multiwalled carbon nanotubes (MWCNTs), were studied. The STF's viscosity increases abruptly during impact tests. The addition of a small amount of carbon nanotubes (CNTs) to the STF, leads to an increase of the maximal viscosity from 2128 to $12,213 \mathrm{~Pa} \cdot \mathrm{s}$. To show the differences between various compositions, the microstructures of fluids were observed by scanning electron microscopy. A pronounced influence of the CNTs on the ability of impact force absorption was noticed. The protective structure containing 55 and 0.25 vol\% of fumed silica and CNTs, respectively, is able to absorb up to $74 \%$ of impact force.
\end{abstract}

Keywords. Shear thickening fluids; rheology; carbon nanotubes; fumed silica; force absorbing systems.

\section{Introduction}

Shear thickening fluids (STFs) can be used as a component of structures with high energy absorbing efficiency [1-3]. This possibility results from the fact that the viscosity of the STF increases abruptly with an increase of shear stress. In the case of sudden strike, STFs change properties from being viscous to elastic, providing a rigid, protective layer. This change is fully reversible - after the strike, the STF comes back to the liquid state. STFs are promising for various applications, such as liquid body armour [4,5], sport protectors, such as smart knee pads [6] and others. The STFs consist of suspensions of solid phases in a carrier liquid. Polyethylene or polypropylene glycols and nanoparticles of fumed silica are mostly used for the fabrication of STFs $[7,8]$. STFs can also contain some additional modifications. The reported values of the maximum viscosity of modified STFs are $600 \mathrm{~Pa} \cdot \mathrm{s}$ for STFs with $\mathrm{SiC}$ nanowires [9], $265 \mathrm{~Pa} \cdot \mathrm{s}$ for STFs modified with calcinated colloidal silica microspheres [10] and $168 \mathrm{~Pa} \cdot \mathrm{s}$ for STFs with graphene oxide [11]. The properties of STFs could also be modified by the addition of graphene nanoplatelets [12].

In this paper, the basic composition of the STF was modified by the minor addition of multiwalled carbon nanotubes (MWCNTs). The rheological properties and impact absorption abilities were measured and discussed.

\section{Methods and materials}

STFs were fabricated by mixing amorphous silica KE-S50, with an average particle size of $500 \mathrm{~nm}$, from Nippon
Shokubai, with polypropylene glycol having a molar mass of $2000 \mathrm{~g} \mathrm{~mol}^{-1}$. The contents of silica were 50,53 and $55 \mathrm{vol} \%$, respectively. The compositions of STFs were modified by the addition of MWCNTs (Nanocyl NC7000, with a diameter of $9.5 \mathrm{~nm}$ and a length of $1.5 \mu \mathrm{m}$ ), with the amounts of $0.05,0.15$ and $0.25 \mathrm{vol} \%$. The relationship between the viscosity and shear rate was determined using an ARES rheometer, equipped with two parallel plates $(\Phi 25 \mathrm{~mm})$, with the gap being $0.3 \mathrm{~mm}$. All the viscosity measurements were performed at room temperature. The solid components, fumed silica and carbon nanotubes (CNTs) were observed using HITACHI SU 8000 and S 5500 scanning electron microscopes (SEM).

The impact protective structures were fabricated by impregnation of polyester three-dimensional woven fabric M8180 from Baltex Ltd. (thickness of $7 \mathrm{~mm}$ and aerial mass of 500 $550 \mathrm{~g} \mathrm{~m}^{-2}$ ) with the STF and sealing it in silicone forms $(130 \times 30 \times 15 \mathrm{~mm})$. The impact tests were carried out using a home-made impact tower, by dropping an impactor, with an energy of $5 \mathrm{~J}$, onto the sample. The procedure is based on British Standard BS 7971-4:2002 'Protective clothing and equipment for use in violent situations and in training. Limb protectors. Requirements and test methods'. The rig for testing of the force absorption consists of a steel anvil and steel impactor that can be dropped on the anvil with required energy. The force sensor, for the registration of the force $v s$. time, is located under the anvil. Initially, the reference test, by dropping the impactor directly on the anvil (without the sample), was carried out. Subsequently the samples, $130 \times 30 \times 15 \mathrm{~mm}$, were mounted on the anvil using tapes with four $2 \mathrm{~kg}$ weights. The impactor was elevated to the position 


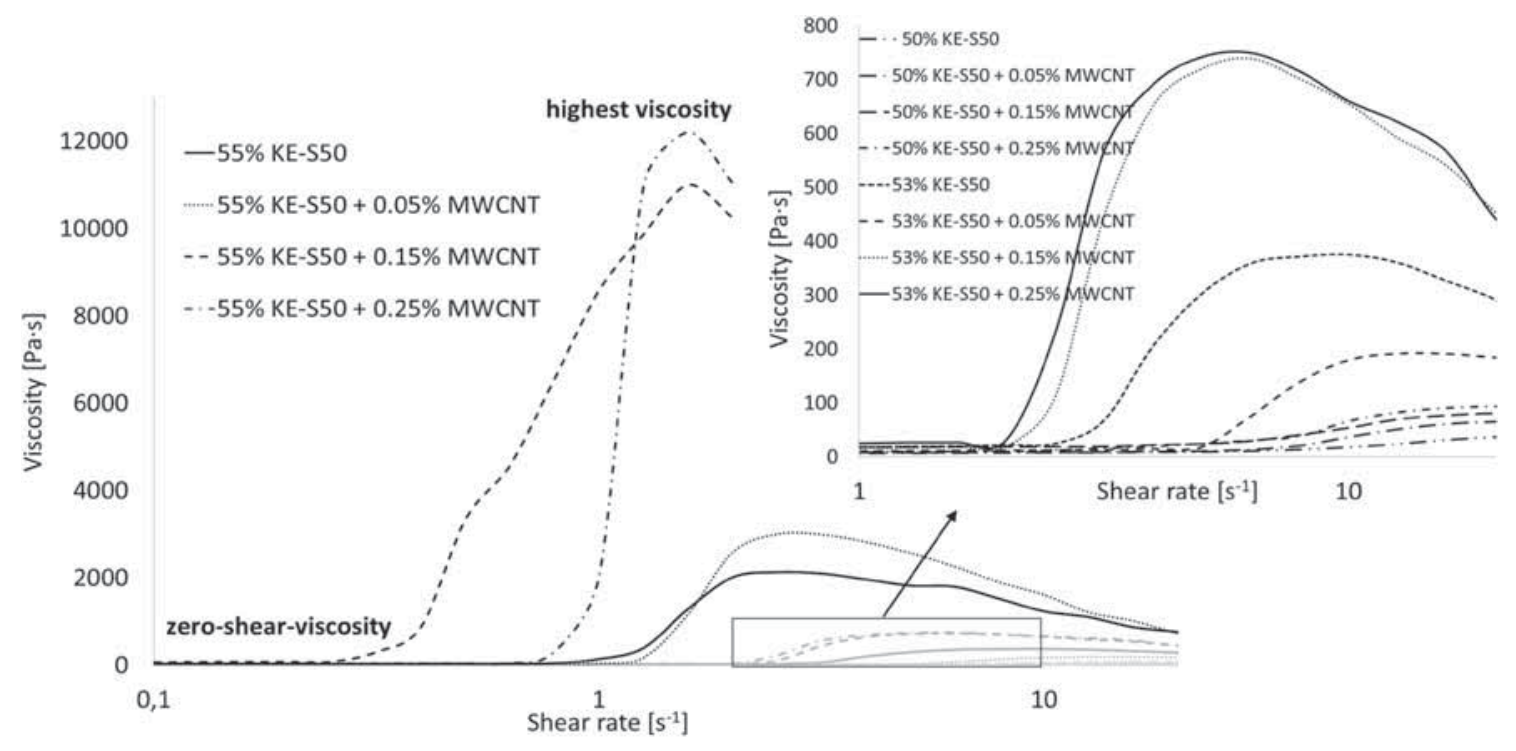

Figure 1. Viscosity vs. shear rate for the STFs with different contents of CNTs.

Table 1. Viscosity and shear rate for the STFs with different contents of CNTs*.

\begin{tabular}{lcccc}
\hline $\begin{array}{l}\text { KE-S50 } \\
(\text { vol\% })\end{array}$ & $\begin{array}{c}\text { MWCNT } \\
(\text { vol\% })\end{array}$ & $\begin{array}{c}\text { Zero-shear- } \\
\text { viscosity (Pa.s) }\end{array}$ & $\begin{array}{c}\text { Highest } \\
\text { viscosity }(\mathrm{Pa} \cdot \mathrm{s})\end{array}$ & $\begin{array}{c}\text { Critical shear } \\
\text { rate }\left(\mathrm{s}^{-1}\right)\end{array}$ \\
\hline 50 & 0 & 13 & 42 & 5.0 \\
& 0.05 & 17 & 66 & 6.3 \\
& 0.15 & 25 & 81 & 4.0 \\
53 & 0.25 & 58 & 94 & 3.2 \\
& 0 & 15 & 375 & 2.5 \\
& 0.05 & 16 & 192 & 3.9 \\
55 & 0.15 & 19 & 740 & 2.0 \\
& 0.25 & 32 & 750 & 1.9 \\
& 0 & 24 & 2128 & 0.6 \\
& 0.05 & 34 & 2996 & 0.8 \\
& 0.15 & 69 & 10,995 & 0.3 \\
& 0.25 & 36 & 12,213 & 0.6 \\
\hline
\end{tabular}

*The experimental error in the viscosity and critical shear rate measurements can be in the range of $1-10 \%$ [13].

providing the impact energy of $5 \mathrm{~J}$ and dropped. The ability of force absorbing was calculated from relationship (1):

$$
E=1-\frac{F_{1}}{F_{2}} * 100 \%
$$

where $F_{1}$ is the force recorded directly for the empty anvil (reference force) and $F_{2}$ is the force recorded for the sample.

\section{Results and discussion}

The values of the zero-shear-viscosity (z-s-v), the highest viscosity and the critical shear rate (shear rate at which viscosity increases abruptly) are presented in figure 1 and table 1 .
The highest viscosity and the critical shear rate at which a drastic increase of the viscosity begins depend on both the concentration of fumed silica and CNTs. The highest viscosities were recorded for the fluids containing $55 \mathrm{vol} \%$ of silica.

Increasing the volume fraction of CNTs results in an increase in the value of the highest viscosity. The STF with $55 \mathrm{vol} \%$ of silica and $0.25 \mathrm{vol} \%$ of CNTs shows the highest value of the highest viscosity $(12,213.3 \mathrm{~Pa} \cdot \mathrm{s})$. This value is almost six times higher than the result for the CNT-free fluid. The critical strain rate initially slightly increases, for $0.05 \%$ CNT, and subsequently, for higher CNT concentrations, it noticeably decreases. The modified fluids also generally have higher zero-shear-viscosity, which means that they can be more efficiently immobilized in fabric spacer. Concentrations of CNTs higher than $0.25 \mathrm{vol} \%$ lead to a drastic increase of the 

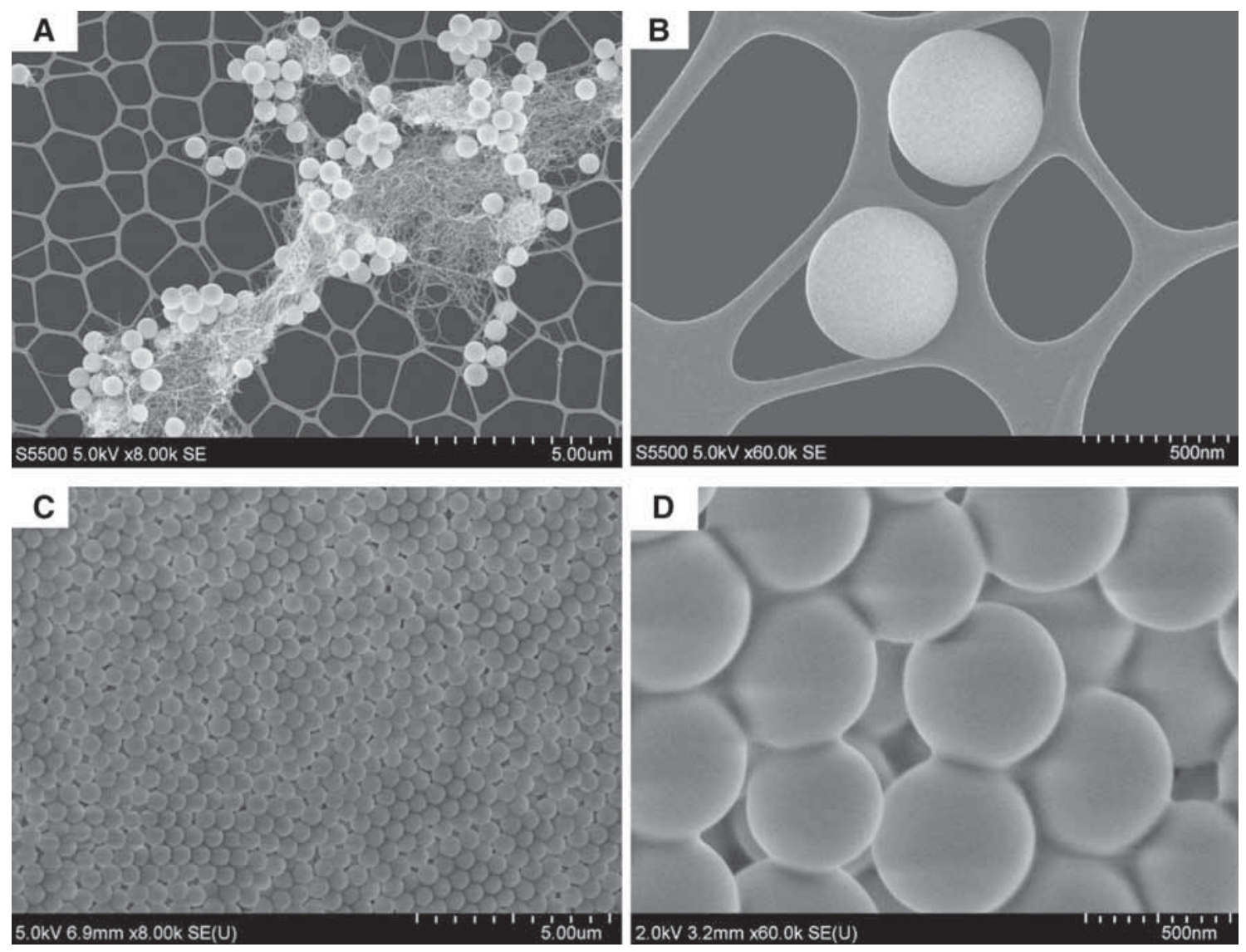

Figure 2. SEM images of (a) the starting mixture of KE-S50 silica and agglomerates of CNTs, (b) image of the KE-S50 silica, (c and $\mathbf{d})$ the arrangement of solid components in the STF.

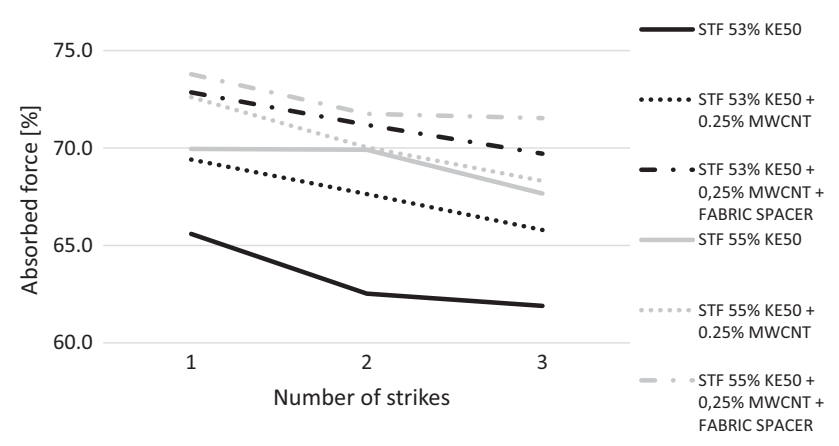

Figure 3. The mean values of absorbed force for STFs sealed in silicone forms after one, two and three strikes.

zero-shear-viscosity. The STF becomes friable and turns into a paste, making impregnation of the fabric impossible.

The solid substrates of the STFs were subjected to SEM observations (figure $2 \mathrm{a}$ and $\mathrm{b}$ ). It can be seen that the silica used for testing has a slight tendency to agglomerate and the particles have a spherical shape (figure $2 \mathrm{a}$ and $\mathrm{b}$ ). In addition, it has a fairly uniform size distribution with low surface development. The CNTs, in contrast, have a huge tendency to agglomerate, forming bundles (figure 2a).

The silica powder and CNTs were mixed before STF fabrication (figure 2a). One can see that CNT agglomerates are covered by small particles of silica. In figure $2 \mathrm{c}$ and $\mathrm{d}$ the distribution of silica and CNTs in the STF is shown. The agglomerates disappeared and only silica particles are visible. We assume that mixing of fumed silica with CNTs in a carrier liquid leads to breaking of the agglomerates of the nanotubes and their even dispersion in the voids between the silica particles, leading to a higher friction in the course of shearing. This is evidenced by the lack of the agglomerates in figure $2 \mathrm{c}$ and $\mathrm{d}$. This fact results in an increase in both the zero-shear-viscosity and the highest viscosity for the STFs containing CNTs, and show better force absorbing ability.

The synthesized STFs were used for impregnation of 3D fabric, which was subsequently sealed in thin silicon forms $(130 \times 30 \times 15 \mathrm{~mm})$ for impact tests. In figure 3 , the average values of the absorbed impact force, after one, two and three strikes in the same place, are shown. One can see that a higher amount of fumed silica provides an increase in the absorbed force. The addition of CNTs causes an additional increase 
in the force absorbing properties. The sample based on STF containing 55 vol\% KE-S50 and 0.25 vol\% CNTs shows the best absorbing properties ( $74 \%$ on the 1 st strike).

The lowest value of absorbed force, for the first strike, was obtained for STF with 53\% KE-S50 (66\%). However, it is also shown that impregnation of the fabric spacer with the STF having $53 \mathrm{vol} \%$ of fumed silica and $0.25 \mathrm{vol} \%$ of CNT results in the force absorbing efficiency of $1.24 \%$ per $1 \mathrm{~g}$ of the sample when compared to pure silica, exhibiting $1.17 \%$ per $1 \mathrm{~g}$ of sample. The mean value of the stability changes is $4.6 \%$. The best stability, which corresponds to a change of only $3.1 \%$, is observed in the sample with $55 \mathrm{vol} \%$ of fumed silica and $0.25 \mathrm{vol} \%$ of CNTs in the fabric spacer. A slight decrease in absorbed force, after the 3 rd strike, is caused by the change in the structure of silicon forms. The STF's structure does not undergo destruction because its properties change by the reversible rearrangement of silica particles in the carrier liquid. One can clearly see that the STFs modified by CNTs have a greater ability to absorb impact force than the ones based on fumed silica only.

\section{Conclusions}

Increasing the volume fraction of the CNTs in the silica-based STFs results in higher $\mathrm{Z}-\mathrm{S}-\mathrm{V}$ and the highest viscosity values obtained in the rheological tests. This is caused by the fact that in modified fluids more particles are in a closer proximity to each other, which results in an increasing internal friction in the samples. Dispersed nanotubes have better opportunities to penetrate the voids within the silica clusters and block the deformation of the suspension.

The improved rheological properties have a positive influence on the impact force absorbing efficiency. The protective structure containing 55 and 0.25 vol\% of fumed silica and CNTs, respectively, is able to absorb up to $74 \%$ of impact force.

\section{Acknowledgements}

Financial support from the National Centre for Research and Development and National Science Centre, within TANGO programme, agreement No. TANGO1/269556/NCBR/2015, project: 'Application of non-Newtonian fluids in energy absorbing systems' is greatly acknowledged.

\section{References}

[1] Gürgen S 2017 Prog. Polym. Sci. 7548

[2] Leonowicz M, Kozłowska J, Wierzbicki Ł, Olszewska K, Zielińska D, Kucińska I et al 2014 Fib. Tex. 2228

[3] Leonowicz M, Kozłowska J and Wierzbicki Ł 2014 Appl. Mech. and Mat. 44013

[4] Wierzbicki Ł, Wilbik-Hałgas B, Struszczyk M and Leonowicz M 2014 Tex. Res. J. 841569

[5] Majumdar A, Butola B and Srivastava A 2013 Mat. Des. 51 148

[6] Li W, Ding J and Shen S 2011 Rec. Pat. Mat. Sci. 443

[7] Singh M and Verma S 2018 Mat. Res. Ex. 55

[8] Moriana A D and Tian T 2016 Korea Aust. Rheol. J. 28197

[9] Ge J and Tan Z 2017 Res. in Phys. 73369

[10] Zheng S 2015 Smart Mater. Struct. 2485

[11] Huang W, Wu Y, Qiu L, Dong C, Ding J and Li D 2015 Adv. Cond. Matter Phys. 20155

[12] Sha X and Kejing Yu K 2013 J. Nanopart. Res. 151816

[13] Laun M, Auhl D, Brummer R, Dijkstra D, Gabriel C, Mangnus M et al 2014 Pure Appl. Chem. 861945 